\title{
Acceptability and use of female condoms among young people in South Africa
}

\author{
Nomsa Mahlalela ${ }^{1}$, Pranitha Maharaj ${ }^{2}$ \\ University of KwaZulu-Natal \\ Durban \\ 4041 \\ Maharajp7@ukzn.ac.za
}

\begin{abstract}
The female condom was introduced more than two decades ago and remains the only female-initiated tool available that provides dual protection for women. The aim of this study to explore acceptability and potential demand for the female condom in Durban, South Africa. The study draws on 15 in-depth interviews with young women aged 18 to 30 years who reported that they had ever used the female condom in the past. The findings highlight a number of factors that facilitate and inhibit female condom use. Protection from sexually transmitted infections (including HIV) and pregnancy prevention facilitates the use of the device. In addition, students expressed positive attitudes towards the female condom and prefer it over other contraceptive methods because it offers them dual protection. Absence of side effects, and greater power and autonomy to initiate safer sex are other factors that facilitate use. Inadequate availability, insertion difficulties, and stigma serve as significant barriers to consistent female condom use.
\end{abstract}

Keywords: Female Condom Use, Acceptability, Young Women, South Africa.

\section{Introduction}

South Africa has one of the highest HIV prevalence rates in the world and the largest number of people living with AIDS (Shisana et al., 20I4). Particularly disturbing is the high levels of HIV infection among women and particularly young women. According to the latest national population survey, the level of HIV infection is higher among young women than men (Shisana et al., 20I4). In South Africa early childbearing is also of major concern, with almost one third of teenagers having been pregnant and the majority of these pregnancies are unplanned (Willan, 2013). Because of their low social status, women and girls often lack decision-making power in sexual relationships and they are rarely able to choose when to have sex, how many children to have or whether to use contraception or protection against sexually transmitted infections (STIs). Limited access to economic resources and the threat of violence force many women to yield control over sexual relations to men. In this context, the female condom remains a valuable tool that provides dual protection against the risk of pregnancy and STIs including HIV/AIDS.

In the 1980s the female condom was introduced to empower women to practice safer sex. It is the only female-initiated barrier method that provides dual protection against the risk of pregnancy and sexually transmitted infections (including HIV/AIDS).

In South Africa, studies suggest that the uptake of the female condom use is low (Bekinska et al. 20I2;
Peters et al. 20I4; Seutlwadi, 20I2). A cross-sectional population-based household survey conducted in four provinces in South Africa found that only $6 \%$ of women who reported currently using a contraceptive method were using the female condom. Research suggests that there are many complex factors influencing individual's conceptive decision-making and sexual behaviour is shaped by existing social and cultural forces including women's status in society (Bekinska et al., 2012; Peltzer et al., 2010; Marston and King, 2006; Seutlwadi, 20I2).

A review of the literature suggests that the use of female condoms is effective in increasing the number of protected sexual acts and reducing the risk of sexually transmitted infections (STIs) (Hoffman et al., 2004). In addition, a choice of a broader range of prevention methods is an important motivating factor in achieving safer sexual practices (Peters et al., 20I4). 2009). Hoffman et al. (2004) suggests that the female condom provides women with an opportunity to take part in sexual negotiations rather than leaving all sexual decisions in the hands of men. In addition, women view the female condom as a means of enhancing safer sex bargaining power within the relationship. Female condoms can contribute greatly to the improvement of reproductive health outcomes and to protecting the health and rights of women. However, studies suggest that female condom users may encounter similar problems to those of male 
condom users. Acceptability studies of the female condom found that the specific concerns of women relate to physical appearance, difficulty with insertion and cost of the device (Beksinska et al., 2012; Mathenjwa \& Maharaj, 20I2).

Recently, there has been a growing number of studies on female condom use. However, much of the research has tended to be quantitative, not fully addressing factors facilitating and inhibiting female condom use. The influence on female condom use is likely to be myriad and varied. Understanding the various factors that influence female condoms could potentially facilitate greater support for this device. This study attempts to understand the complex factors that could potentially facilitate greater support for this devise. It looks more specifically at the factors facilitating and inhibiting female condom use. Interviews were conducted with young African female students aged 18 to 30 years. In Africa, research suggests that the university is a high-risk institution for the transmission of HIV because of high-risk activities such as 'sugar-daddy' practices and unprotected casual sex with multiple partners (Njagi \& Maharaj, 2006; Mantell et al., 20II). Though far from typical of young people, however, they are of particular importance for the future of the country and lessons can be learnt from this group that may have relevance to the wider population of young people.

\section{Methodology}

The study draws on qualitative study using in-depth, open-ended interviews. In total, 15 interviews were conducted with female university students. The small size of the sample is related to the difficulty in locating ever users of female condoms. However, the size of the sample is not a problem given that the main focus of the study is not generalizability but to better understand acceptability of female condoms.

\section{Setting and Sampling}

Interviews were conducted with students at a public university in Durban. Durban is situated in the province of KwaZulu-Natal and remains one of the heavily populated cities in the country. According to Statistics South Africa (2007), approximately 3 million people are residing in this city and the majority of the population are Africans followed by Indians, Whites and Coloureds. The province has one of the highest HIV prevalence rates of HIV/AIDS in the country, and is confronting major sexual and reproductive health challenges (Health Systems Trust, 2009).

Participants were recruited through the campus clinic which holds the records of all students requesting female condoms. The head of the clinic was contacted and provided with written information about the study to disseminate to students within their respective networks. Interested students were approached by the researcher to arrange an interview. Through snowball sampling, additional women were recruited to the study. Each participant was given information about the purpose of the study and informed consent was obtained from each woman before the beginning of the interview.

The study was restricted to young women who had ever used the female condom. Ever users include current and former users. Current users were defined as those who reported using the female condom and they had used it at least in the last sexual intercourse and former users were defined as those who reported using the female condom but they were no longer using it at the time of the study. The study focused on female students because the focus of the study is on a female initiated device. The study was limited to ever users (current and former) of the female condom to identify potential facilitators and inhibitors given their experience with the device. In addition, only those who were aged 18 years and over were eligible for the study. Female students 18 years and older were chosen for this study because this is the legal age in South African law where a person is considered an adult and is fully capable of acting independently and having sex at this age is not illegal (Strode, Slack and Essack, 2010).

\section{Interview Process}

An interview schedule was used which remained the same for each interview, but was open-ended and allowed the interview to probe for further clarification. Each interview began by assessing the demographic profile and contraceptive history of each participant. Questions covered included knowledge of female condoms, sources of information about the female condoms, reasons for use, as well as barriers to use. All of the interviews were conducted onsite at the university at a venue that ensured maximum privacy. Each interview lasted on average about 45 minutes. All interviews were digitally recorded. Interviews were conducted in English by the principal investigator.

\section{Ethical Approval}

Ethical approval was obtained from the Ethics Committee at the University of KwaZulu Natal. At all times, confidentiality was maintained and participants were aware that no identifiers will be available in the report. All participants were assured that their participation in the study was voluntary and they were free to withdraw from the study at any time. 


\section{Analysis}

All the interviews were recorded with the permission of the women. The recorded interviews were transcribed and analysed using thematic analysis. Thematic analysis involves categorising the data into particular themes that arise on more than one occasion in the interviews. Verbatim quotes from the interviews are used to illustrate particular findings.

\section{Results}

The ages of the women ranged from 22 to 27 years old. Almost all the women were Zulu speaking with the exception of two women. All participants were currently sexually active and ever-users of the female condom. Of the women interviewed, II were currently using the female condom and four were former users. The interviews with the women reveal that female condom use is not consistent. The majority of the women $(n=8)$ reported that they do not use the device consistently.

\section{Sources of Information}

The interviews revealed that female condoms are not well advertised and as a result the young women reported that they did not receive detailed information about it. More than half of the women stated that they had found out about the female condom on campus. Most of these women said that they heard about the female condom at the health centre and they decided to get information about it. They also noted that the female condom was not well advertised so they found it difficult to get information about it.

"I found out about the female condom on campus. Some nurses from the clinic came to campus to promote the female condom and they gave us samples and that is when I found out about this product and I actually took one home" (P3).

\section{Factors facilitating use}

Perception of risk is an important motivating factor for using the female condom. The women perceived themselves at high risk of infections and pregnancy and this led to them using the female condom.

"After hearing my lecturer speak about the femidom and all the reasons she gave on the use of female condoms. I thought that it would be better for me to use it as well to protect myself against HIV infection and other infections that occur in sexual intercourse and to prevent pregnancy even though we know that it is not $100 \%$ protective but then it is something" (P/3).
"I used the female condom mainly because I wanted to prevent myself from getting diseases like AIDS, STDs and also to prevent myself from getting pregnant" (PI4).

Some women prefer the female condom to other methods of contraception. They value the dual benefits of female condoms. The women were aware that hormonal contraceptive methods provide no protection against the risk of HIV. In addition, they observed that the female condom does not have negative side effects associated with hormonal contraceptive methods including weight fluctuations, irregular bleeding, increase in acne, and decrease in libido.

"I believe in natural things so for me using all these other measures for preventing pregnancy are not sufficient and are not healthy and they have side effects. Also I think the female condom is more safe and more protective because it helps you prevent HIV as well, unlike the other measures of contraception" (PIO).

"Well, you can use contraceptives like pills but they will never protect you from contracting HIV and other STIs. They do not work $100 \%$, you find that there are people who use pills but they still get pregnant. It is rare but it happens. But with the female condom you are sure that there would not be any pregnancy and you would not contract any other STIs if it is used consistently and correctly" (P5).

In South Africa dual method use is not common. Dual method use is the simultaneous use of condoms with another method of contraception. There was however one woman who reported using dual methods: the condom in combination with the injection. She emphasized that she used two methods in order to minimise the risk of pregnancy and HIV/AIDS.

"I do not use female condoms only. I also use other prevention measures like injection. It is 'double protection' if I can say" (P8).

Women also reported that sexual experimentation with the device was the initial reason for using this method. They stated that they experimented with the female condom in order to see how it works. This was mainly because they were not as familiar with the female condom as the male condom. 
"To be honest the main reason why I used the female condom was to just try it out and see what this thing is about. When we think about condoms we only think about the male condom. I decided to try it out and see what it is all about" (P7).

Of course in this life things are very fast and you want to protect yourself. The way the lady has described the female condom to me, I felt the need to use the female condom because I wanted to try it so that was the reason" $(\mathrm{PI})$.

Women also pointed out that this method gives them the opportunity to protect themselves in their sexual relationships especially if their partner is not willing to use protection. It improves their negotiating power in sexual relationship. The women pointed out that they can give their partners the option to choose from the male and female condom. In addition, they can use the female condom when their partner gives an excuse for refusing to use the male condom. Therefore, this results in an increased number of protected sexual acts because if the male partner is not willing to use protection then the woman will use the female condom. For them, the female condom gave them greater control over their own bodies and sexual lives.

"As I just said that it is empowering to know that as a woman you get to decide to use the condom, like normally it is men that uses the condom. For me personally, I also believe that the female condom gives power to young women like me regarding my sexual health. It gives control over my own body and a sense of control over my sexual life" (P2).

"Because sometimes men usually do not carry condoms around them. So in order to be on the safe side it is better if I carry my own, so whenever he uses an excuse that he does not have the condom so we will not have unprotected sex, I would just take my own from my bag" (PII).

"Well, I would think the reason for using a female condom is because as a lady you would like to be in charge of a situation that could put you or your health in danger. So I feel that as an independent woman you are just taking charge of the sexual situation" (PI2).

Fidelity in sexual relationships is important but the women stated that it is not possible to completely trust someone, thus it was important for them to always protect themselves. They reported that it was important for them to be always on the safe side because in as much as they love their partners they could not be completely sure that their partners are faithful to them. Distrust between partners is common in relationships and is one of the significant factors facilitating the use of protection. One woman explained that she uses the device with her partner because she does not trust him. She further highlighted that her partner is always out with other women, and this has forced her to have many female condoms in her room in order to protect herself.

"Even though you can be in a relationship or in a committed relationship but you can never be sure especially in this country, so it is important to protect yourself. If you are in a relationship where your partners says he loves you, then he should love you enough to protect you" (P7).

"Because as much as I love him I cannot write it in blood that I know what he is doing with his life. So it is just important for me to be on the safe side" $(P / 2)$.

\section{Factors inhibiting use}

There are a number of barriers that prevent women from using the female condom consistently. Women reported experiencing difficulties with the initial use of the device. They reported that when they started using the device they experienced difficulties in inserting and properly positioning the female condom. The women pointed out that even though the female condom comes with instructions, they are not easy to follow. This method is hardly promoted and available, and it is very rare to find pamphlets advertising the device. Women noted that they experienced difficulties inserting the device the first time they used it. One woman stated that inserting the device was a "nightmare". However, they admitted that the device becomes easier to use with practice.

"It is weird inserting it. It is like you are putting on a tampon and if you are not a person who uses a tampon it is very difficult to insert the female condom when you are the first time user" (P2).

"The major problem was actually at the beginning when I first started to use the female condom. I had a bad experience at first when I tried to insert it, I read the instructions but it was not easy. The practical part of actually applying it was a "nightmare" I had to do it over and over again" (P6).

Women also reported that the female condom sometimes slips off and this reduces the efficiency of the device. They felt that it was difficult to properly 
position the female condom and this resulted in them no longer using the method. The female condom must be positioned properly by ensuring that the inside ring of the device is attached tightly to the cervix.

"Ok, sometimes it falls off. It does this if it is not inserted properly. The other day it fell off. (PII).

Women also reported that it is difficult to access the female condom. They reported that compared to the male condoms, female condoms are not widely available. The device is not available in most public places where you are likely to find the male condom. In addition, female condoms are not as widely promoted as the male condoms.

"I think the supply of the female condom is not as much as male condoms so they are very rare and hard to get" (PI).

"You cannot even find them like in toilets. Yes, even here in the University you can only find the male condom but not the female condom. I think also maybe it is because it is expensive. Maybe this is the reason that you cannot access the female condom easily" ( $p 8)$.

Stigma is another barrier to the use of female condoms. Women reported that female condoms are associated with lack of trust and this is a major barrier to use. Female condoms are associated with illicit sex and promiscuity and women who suggest use are seen in a negative light.

"There is the stigma that is attached to the female condom. As a woman if you talk about it or discuss it with your partner you will find that they are taken like they are a bit forward when it comes to sexual orientation you know. So some women are taken to be promiscuous if they tell other people that they are using the female condom. So basically there is still a lot of stigma attached to the female condom" (P6)

Another problem with the female condoms is that insertion needs to take place before sexual intercourse and the women complain that it makes it appear like they planned on having sex. The women argued that it destroyed the spontaneity of the moment.

"I think the fact that you have to put it on before you can sleep with a person is a "turn off". Maybe future developers of female condom should create something that you do not have to wait before you can use it like the male condom. That should give you the freedom to be spontaneous" (P2).
"Because you need to insert it before you actually have intercourse, you know that you are about to have sex. It is not comfortable because sometimes when you are about to have sex with a person and it is unplanned, it just "kills the vibe" and "kills the romance" because it is like you actually planned the whole process, it does not happen naturally" (PII).

\section{Discussion}

More than twenty years have lapsed since the female condom has been introduced around the world. The device remains the only available female initiated tool that provides double protection against the risk of pregnancy and HIV/AIDS. While most studies have focused on the prevalence of female condoms this study attempts to provide insights into the acceptability of female condoms and factors affecting potential demand. Of course, the data is not generalizable to the entire population because they are based on a relatively small sample; in addition, university students constitute an elite sector of the population, as most of the population of South Africa does not have a tertiary qualification. Nevertheless, several key issues emerged from the results.

Several factors influence acceptability and use of female condoms. The women expressed positive attitudes to female condoms. They felt safe and protected when using the female condom. They were particularly aware of the dual protective benefits of the female condom. Female condoms protect not just against pregnancy but also sexually transmitted infections (including HIV/AIDS). For many young people, peers are an important source of information. However, most of the women reported that they had obtained information about female condoms from health facilities rather than friends.

Individual perception of risk has been identified as a significant factor influencing adoption of safer sexual practices, such as the use of condoms. The study found that perception of risk was instrumental in influencing their decision to use female condoms. Women perceived themselves at risk of pregnancy and HIV/AIDS and this motivated them to try the female condom. However, perception of risk alone is not sufficient to translate into use of female condoms. Other factors also influence use of female condoms. The device is a female-controlled tool which was developed to provide women with an independent method without relying on their male partners. In this study the women agreed that this method gave them a greater sense of power and control in their sexual relationship. It was clear that the women viewed the female condom as improving their decision-making 
power in sexual relationships. The women felt that this method provides them with an alternative strategy especially if their partner does not want to use protection. Thus, the female condom provided them with an opportunity to participate actively in reinforcing safer sex in relationships as they get to decide on female condom use. They further highlighted that the device gives power to young women like them regarding their sexual health. Meaning that they felt they have control over their sexual health. Furthermore, women felt that with the availability of the device they are able to give their partners an option between the female and the male condom whenever their male partner provides an excuse for not using protection.

Women also preferred the female condom to other methods of contraception. The change in the body image that is associated with other methods was highlighted as a barrier to the uptake of these contraceptive methods. In general, using injections as a contraceptive method is associated with major side effects such as weight gain, irregular bleeding and loss of libido. Across all the interviews the women reported that they will not stand for the negative side reactions of hormonal preventative measures and this became an important facilitator of female condom use because the device does not contain any wellknown negative side reactions. Therefore, the female condom was found to be a best option to hormonal contraceptives by the women and this was emphasized by almost all the participants who had either experienced the side effects or were fearful of the side effects.

The female condom is a fairly unique contraceptive method in terms of its double protection function against STIs including HIV and unwanted pregnancy. However, the device is not without challenges like any other contraceptive methods. A number of women reported a number of barriers that has led to them not using the condom consistently. The women reported that the first time they used the device they experienced difficulties in inserting and properly positioning it. For instance, some mentioned that they were unsure how much to leave at the bottom and how far to push it up. Their major concern was that even though the female condom comes with all instructions however the practical part of inserting it for the first time is a "nightmare". Other studies have also reported similar difficulties (Mathenjwa and Maharaj, 20I2). Nevertheless, the women also acknowledged that with practice the method becomes easier to use. Also vaginal irritation and slippage was reported by the women in this study and it was linked with less motivation to use the device. This is consistent with other studies (Sarkar, 2008) which revealed the discomfort of the device as an important barrier to its use. There were also misconceptions reported by a few women. A few women stated that the female condom needs to be inserted a couple of hours before sexual intercourse. However, this is not true because the device can be inserted just before sex like the male condom. It is important to address such misconceptions about the device because they perpetuate stereotypes which inhibit female condom use.

Inadequate availability of the female condom remains an important barrier to female condom use. The women observe that female condoms are not easily accessible. They pointed out that the device is not widely available in stores, hospitals and clinics. This is also supported by Seedat (20II) who argues that despite the introduction of the device over a decade ago, however its supply and utilization remains insufficient. Nakari and Huurne (2010) assert that female condom distribution in sub-Saharan Africa is inadequate with one in every 300 women having access to the device. Therefore, this shows that despite that the device has been in existence on the market for a long time; however, the availability remains inadequate and as a result, underutilized. According to Peters et al. (20|4), inadequate investments from donors and the lack of confidence from the government's side hinder the widespread distribution of the device.

Stigma associated with female condom use was also highlighted as a barrier to the use of the device. Women reported that in general condoms are associated with lack of trust and in most cases are seen to be suitable for use in casual partnerships especially commercial sexual encounters. Sarkar (2008) further asserts that due to the way in which they are promoted in the media, condoms are further linked to promiscuity. Therefore, women find it difficult to use female condoms when they are crowded by such negative perceptions. Such findings correspond with results from other studies that found that residual social stigma attached to the condom was associated with less frequent use of condoms (Seutlwadi., 2012; Sarkar, 2008).

The study suggests that although female condom use involves negotiation with their partners, the fact is that it offers women an independent, additional method of protection that gives them more power and also, increases their ability to control their sexual and reproductive health. However, there is a need for greater availability of the female condom. 


\section{References}

Beksinska M, Smit J, Mantell J. 20I2. Progress and challenges to male and female condom use in South Africa. Sexual Health, 9, (I): 5I-58

Health Systems Trust. 2009. Health Statistics: HIV prevalence (\%). Durban: Health Systems Trust.

Hoffman, S., Mantell, J., Exner, T., \& Stein, Z. 2004. The future of the female condom. Perspectives on Sexual and Reproductive Health, 36, (3): I20-I26.

MacPhail, C., Pettifor, A. E., Pascoe, S., \& Rees, H. V. 2007. Contraception use and pregnancy among 15-24 year old South African women: a nationally representative cross-sectional survey. Bio Medical Central Medicine, 5, I-8.

Mantell, J. E., Smit, J. A., Jennifer, A., Beksinska, M., Scorgie, F., Milford, C., Balch, E., Mabude, Z., Smith, E., Adams-Skinner, J., Exner, T.M., Hoffman, S., \& Stein, Z. A. 20II. Everywhere you go, everyone is saying condom, condom. But are they being used consistently? Reflections of South African male students about male and female condom use. Health Education Research, 26, (5), 859-87I.

Marston, C., \& King, E. 2006. Factors that shape young people's sexual behaviour: a systematic review. Evidence Based Nursing, 10, (4), 124.

Mathenjwa, T., \& Maharaj, P. 20I2. Female condoms give women greater control': a qualitative assessment of the experiences of commercial sex workers in Swaziland. European Journal of Contraceptive Reproductive Health Care, 17, (5), 383-392.

Nakari, T., \& Huurne, D. 2010. The condom gap, widening or narrowing? IPPF.

Sarkar, N. N. 2008. Barriers to condom use. The European Journal of Contraception and Reproductive Health Care, 13, (2), I I4-122.
Njagi, F., Maharaj, P. 2006. Access to Voluntary counseling and testing services: Perspectives of young people. South African Review of Sociology, 37, (2): I I3-I 27.

Peltzer, K., Mzolo, T., Mbelle, N., Tsoai, L., Lewa, N., \& Ncitakalo, N. 20I0. Dual protection, contraceptive use, HIV status and risk among a national sample of South African women. Gender and Behaviour, 8, 2833-2845.

Peters, A., Van Driel, F. \& Jansen, W. 2014. Acceptability of the female condom by subSaharan African Women: A Literature Review. African Journal of Reproductive Health, 18, (4): 34-44.

Seedat, F. 20II. What is hindering female condoms from preventing HIV transmission? The progress of policy thus far. Consultancy Africa Intelligence

Seutlwadi, L. 20I2. Contraceptive use and associated factors among South African youth: A populationbased survey. South African Journal of Obstetrics and Gynecology, I8, (2): 43-47.

Shisana, O., Rehle, T., Simbayi, L.C., et al. 2014. South African National HIV Prevalence. Incidence and behaviour survey, 2012. Cape Town: HSRC Press.

Statistics South Africa. 2007. Community Health Survey 2007: Basic Results- Municipalities. Statistics South Africa: Pretoria.

Strode, A., Slack, C., \& Essack, Z. 2010. Child consent in South African law: implications for researchers, service providers and policy makers. South African Medical Journal, 100, 247-247.

Willan, S. 2013. A Review of teenage pregnancy in South Africa-experiences of schooling, and knowledge and access to sexual \& reproductive health services. Partners in Sexual Health. 